\title{
A Systematic Review and Meta-analysis of Effect of Probiotic Supplementation on Age-related Sarcopenia in Elderly Adults
}

\author{
Sahar Saraf-Bank \\ Isfahan University of Medical Sciences \\ Fatemeh Navab \\ Isfahan University of Medical Sciences \\ Mohammad Hossein Rouhan \\ Isfahan University of Medical Sciences \\ Zahra Hajhashemy \\ Isfahan University of Medical Sciences \\ Nafiseh Shokri-mashhadi ( Nafiseh.shokri@yahoo.com) \\ Isfahan University of Medical Sciences
}

\section{Research Article}

Keywords: sepsis, continuous veno-venous hemofiltration, iron metabolism, hepcidin, IL-6

Posted Date: December 29th, 2021

DOI: https://doi.org/10.21203/rs.3.rs-1176764/v1

License: (c) (i) This work is licensed under a Creative Commons Attribution 4.0 International License. Read Full License 


\section{Abstract}

Background: Several clinical studies emphasized the role of bio-diversities of gut microbiota in age-related disorders. Nevertheless, the effect of probiotic administration on sarcopenia indices are unclear. This meta-analysis aimed to investigate the effect of probiotic administration on muscle strength, muscle mass, and muscle function.

Methods: We assessed all interventional studies through different electronic databases including PubMed, Scopus, ISI - Web of Science, and Cochrane library using defined keywords from inception to Jun 2021. Studies that investigated the effect of probiotic administration on at least one of the components of sarcopenia or anthropometric indices versus non- probiotics in old adults (>55 years) were included.

Results: The initially overall effect of meta-analysis on 1393 participants declared a null effect of probiotic supplementation on main outcomes, including muscle mass (WMD: -0.05, 95\% Cl: -1.54, 1.43; I-square: 0.0\%, P=0.985), and muscle strength (WMD: 0.7, 95\% Cl: -0.01, 1.40; I-square: 76.8\%, $\mathrm{P}=0.005$ ). Subgroup analysis showed that administration of probiotic supplementation for more than 12 weeks significantly increased muscle strength (WMD: $1.15,95 \% \mathrm{Cl}: 0.86,1.43$; I-square: $0.0 \%, \mathrm{P}=0.679$ ). However, probiotic supplementation had no effect on anthropometric indices, including body mass and body mass index, (WMD: $-0.05,95 \% \mathrm{Cl}:-2.57,1.56$; I-square: 0.0\%, $\mathrm{P}=0.976)$ and (WMD: 0.08, 95\% Cl:-0.16, 0.32; I-square: 0.0\%, $\mathrm{P}=0.718$ ), respectively.

Conclusion: This study confirmed the positive impact of probiotic supplementation on the muscle strength (based on the last definitions by EWGSOP), in particular, probiotic administration for more than 12 weeks. More clinical trials on sarcopenic elderly subjects are wanted to confirm our findings.

\section{Introduction}

Advancement in medicine has led to an increase in global population aging [1]. The percentage of people over 60 years will approximately duplicate from 12-22\% between 2015 and 2050, which is associated with a notable increase in age-related disorders such as sarcopenia and frailty [1, 2]. According to the last definitions by EWGSOP, diagnosing sarcopenia is identified with low muscle strength, and it is considered severe if low muscle strength, low muscle mass, and low physical performance are detected [3]. Poor muscle strength is in association with poor quality of life, risk of falls, fractures, and higher healthcare costs[4]. Despite a growing interest to ameliorate this condition, there is a lack of adequate knowledge underlying the pathogenesis of sarcopenia and an effective therapeutic approach [5]. In this respect, a major challenge would be to recognize the factors protecting muscle health across the lifespan.

Epidemiologic observations indicated that changed gut microbiota structure according to diet, taking drugs, and other environmental factors across the life course lead to distinctive microbiota configurations, composition, and function in the elderly [6, 7]. The gut microbiota has the fundamental role in maintaining many aspects of health[8]. Reduction of microbiota bio-diversities are associated with metabolic changes, physiologic dysregulation, and markers of inflammation that result in age-related adverse health outcomes[9]. For this reason, researchers have hypothesized that gut microbiota composition may have a great relationship with age-related modifications in skeletal muscle mass and function [9]. Recently, experimental studies revealed that changes in the gut ecosystem using probiotics intake affect the muscle function and the inflammatory status of the aged animal models [9-12]. Therefore, examining the effect of probiotic administration in the context of sarcopenia is of particular interest in several clinical trial trials [1316]. However, the clinical outcomes are inconsistent, and there is not a systematic or meta-analytical study that focuses precisely on the effect of probiotic supplements on sarcopenic components. The main aim of this systematic review and meta-analysis was to distinguish the impact of probiotic supplements on age-related sarcopenic components. Moreover, we assessed whether probiotics administration effect on anthropometric indices in old population?

\section{Methods And Materials}

\subsection{Literature research and data sources}

Current systematic review was carried out in compliance with the PRISMA statement[17]. Two investigators (N. Sh-M and F. Navab) independently conducted an electronic literature search using some reliable databases, including MEDLINE (https://www.ncbi.nlm.nih.gov/pubmed) (PubMed), SCOPUS (https://www.scopus.com), ISI -Web of Science and Cochrane library without any restrictions on language or date in order to identify the effect of probiotics on sarcopenia components when compared to standard care or placebo in elderly population (From inception to Jun 2021). The electronic search strategy was done using the following Medical Subject Headings (MESH) and non-MESH keywords: (Sarcopenia OR "Muscle strength" OR "Hand

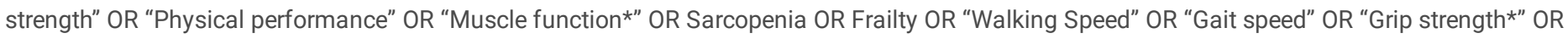
"Hand grip*" OR "lean body mass" OR "Percentage of body fat" OR “Knee extension strength*”) AND (Probiotic* OR "Escherichia coli" OR Microbiota* OR Bifidobacterium OR Lactobacillus OR Saccharomyces OR kefir OR Yogurt) (Supplementary file 1). To expand our research, we separately assess the impact of probiotics on anthropometric and body composition ("Quetelet Index" OR "Body Mass Index" OR "Body weight" OR "calf circumference" OR "Waist circumference*" OR "Body composition" OR BMI OR "skin-fold thickness" OR "fat free mass" OR "body mass") when compared to standard care or placebo in elderly population. The references pointed in the retrieved articles were also searched manually.

\subsection{Inclusion criteria}


Through abstract reading, all studies with the following criteria were eligible to be included in the present review: (1) were interventional studies; (2) compared the effect of administration of probiotics alone as a supplementation versus non- probiotics on at least one of the components of sarcopenia (muscle strength, muscle mass, muscle function) in older adults (mean/median age of 55 years and older). Investigations were not included if they: (1) were duplicated publications; (2) were not conducted on old adults ( $\geq 55$ ); (3) were observational studies; (4) were done on animal models or in vivo studies; (5) were review or protocol articles; (6) were classified as gray literatures, for instance, conference abstracts, government reports and theses. To define the impact of probiotics on body composition, we considered all interventional studies that investigate the effect of supplementation with probiotics on at least one of the anthropometric measurements.

\subsection{Exclusion criteria}

Studies were eligible to be excluded in the present review if: (1) the intervention included whole food or component of foods (such as dairy products) and did not report the dosage of probiotics (2) reported the effect of multiple nutrients along with probiotics supplementation (multi- supplement); (3 The study were conducted on adults (< 55 years); (4) did not report the predefined endpoints as mean ( \pm SD) for sarcopenia components (Supplementary file 2). After abstract reading, all studies, which assessed the effect of probiotics administration alone versus non- probiotics in old adults ( $>55$ years) were included in this review. Additional outcomes were the data related the effects of probiotics on anthropometric and body composition in elderly adult which searched separately with predefined keywords, and results of analysis is reported.

We extracted the following information: author's name, publication year, participants characteristics (sample size, gender, and age), health status, intervention (type of compounds, dose, and duration) and main outcomes, including muscle mass, muscle strength and muscle function. The data were extracted independently by two investigators (F.N, Z.H). We also contacted the corresponding author to obtain the data when necessary (N.Sh-M).

\subsection{Assessment of bias}

Risk of bias assessment: In the current meta-analysis, the Cochrane quality assessment tool was used to examine the risk of bias for each study included[18]. This tool consists of seven domains including reporting bias, detection bias, random sequence generation, allocation concealment, performance bias, attrition bias, and other sources of bias. Each domain was assigned a "high risk" score if there was a methodological flaw that could have affected the findings, a "low risk" score if the domain was not defective, and an "unclear risk score" if no information was available to determine the impact. The overall risk of bias for an RCT was considered: (1) Low; for studies that all their domains obtained "low risk "score, (2) Moderate; for studies that at least one of their domains was given "unclear risk "score, and (3) High; for studies that at least one of their domains was given "high risk". Two independent researchers separately assessed the risk of bias for included studies.

\subsection{Statistical analysis}

The overall effect sizes were computed through the use of mean changes and their SDs of sarcopenia components in groups of probiotic supplementation and control. In cases that mean changes were not reported, changes in values of sarcopenia components during the intervention were considered to compute the mean changes. Furthermore, the method of Hozo et al [19] was used for conversion of standard errors (SEs), interquartile ranges (IQRs) and 95\% confidence intervals (Cls) to SDs. The random-effects model that takes between-study variations into account, was used to obtain the overall effect sizes. In addition, $I^{2}$ statistic and Cochrane's $Q$ test were used to examine heterogeneity. $I^{2}$ value $>50 \%$ or $P<0.05$ for the Q-test was considered as significant between-study heterogeneity. Subgroup analysis was conducted based on categorical confounders such as duration of the intervention ( $\leq 12$ or $>12$ weeks), to explore the probable source of heterogeneity. Additionally, the included studies have reported the muscle mass in two different scales ( $\mathrm{Kg}$ and percentage); so, subgroup analysis was performed for this variable based on scale (Kg and percentage). The extent to which inferences might depend on a particular study was assessed through the use of sensitivity analysis. Visual inspection of Begg's funnel plots and statistical assessment of its funnel plot asymmetry by Begg's test and Egger's test were used to assess publication bias. The STATA version 14.0 (STATA Crop, college station, TX, USA) was used for statistical analyses. P values $<0.05$ were considered statistically significant for all tests including Cochran's Q test.

\section{Results}

\subsection{Study characteristics}

Figure 1 depicted the flowchart of study selection. From different database searching, 1581 articles were detected. After removing duplicate records, 1313 studies were remained for screening. Based on title and abstracts screening, 1275 records were excluded and the full text of 38 studied were reviewed more precisely. Twenty studies were excluded with reasons (supplementary file 2), and then 18 studies were included to the systematic review [20-37]. Regarding meta-analysis, three studies did not eligibility to enter to our analysis. Therefore, only 15 studies were recruited to the meta-analysis $[20,22-24,27-37]$ due to following explanations: First, there was only one study that evaluated the effects of probiotic supplementation on waist to hip ratio (WHR). The results of this study showed a significant reduction in WHR in both probiotic and placebo group [21]. Second, Mane et al. did not report the end point values of BMI. However, it was declared that no significant change was detected in BMI in either probiotic or placebo group [26]. Third, Macfarlane et al. only reported data on weight and had no other anthropometric measurements. No significant difference was reported regarding weight in this study [25].

The characteristics of included studies to the systematic review were presented in Table 1. Totally, 1393 participants were included in the systematic review (older adults more than 55 years). Six studies enrolled healthy participants [21, 22, 25, 26, 31, 37] and other studies recruited people with 
underlying disease, such as obesity, metabolic syndrome, sarcopenia or frailty, and nervous system diseases [20, 23, 24, 27-30, 32-36]. Four studies had duration more than 12 weeks [23, 30,31, 37] and 14 studies had duration less than 12 weeks [20-22, 24-29, 32-36]. In 5 records, foods were enriched by probiotic with certain dosage and applied for intervention group [24, 30, 35-37], and probiotic supplements were used in 13 remain studies [20-23, 25-29, 31-34]. The main strain type of probiotic supplements were Lactobacillus and Bifidobacteria. Most studies administered supplement as probiotic mixtures [20,26, 29, 32-35,38], and one of them used probiotic supplement enriched with oligosaccharides[27]. The nature of placebo in our included studies were water, maltodextrin, cellulose, and potato. 
Table 1

Characteristics of included studies to the systematic review

\begin{tabular}{|c|c|c|c|c|c|c|c|c|}
\hline Author, year & $\begin{array}{l}\text { Sex (F/M), subjects } \\
\text { (Intervention/placebo) }\end{array}$ & Country & $\begin{array}{l}\text { Age } \\
\text { (year) }\end{array}$ & $\begin{array}{l}\text { Type of Intervention } \\
\text { and dosage } \\
\text { (CFU/day) }\end{array}$ & $\begin{array}{l}\text { Type of } \\
\text { placebo }\end{array}$ & $\begin{array}{l}\text { Study } \\
\text { Duration } \\
\text { (weeks) }\end{array}$ & $\begin{array}{l}\text { Health } \\
\text { status of } \\
\text { subjects }\end{array}$ & Measurements \\
\hline \multicolumn{9}{|l|}{ Muscle mass } \\
\hline $\begin{array}{l}\text { Inoue, } 2018 \\
\text { [1] }\end{array}$ & $\begin{array}{l}\text { Female/Male } \\
(20 / 18)\end{array}$ & Japan & 70.3 & $\begin{array}{l}\text { Bifidobacterium/ } \\
1.25 \times 10^{10}\end{array}$ & Water & 12 & Healthy & $\begin{array}{l}\text { Lean body } \\
\text { mass }(\mathrm{kg}) \text { was } \\
\text { measured by } \\
\text { BIA }\end{array}$ \\
\hline Neto, 2013 [2] & $\begin{array}{l}\text { Female/Male } \\
(9 / 8)\end{array}$ & Brazil & 67.9 & $\begin{array}{l}\text { Frutooligossacarid- } \\
\text { probiotic mixture/ } \\
1 \times 10^{9} \text { probiotic }\end{array}$ & Maltodextrin & 12 & Frailty & $\begin{array}{l}\text { FFM }(\mathrm{kg}) \text { was } \\
\text { measured by } \\
\text { BIA }\end{array}$ \\
\hline $\begin{array}{l}\text { Nilsson, } 2018 \\
\text { [3] }\end{array}$ & $\begin{array}{l}\text { Female } \\
(45 / 45)\end{array}$ & Sweden & 76 & $\begin{array}{l}\text { Lactobacillus. } \\
\text { reuteri } 6475 / \\
1 \times 10^{10}\end{array}$ & Maltodextrin & 12 & $\begin{array}{l}\leq 1 \mathrm{SD} \text { for } \\
\mathrm{BMD}\end{array}$ & $\begin{array}{l}\text { Lean body } \\
\text { mass }(\mathrm{kg}) \text { was } \\
\text { measured by } \\
\text { BIA }\end{array}$ \\
\hline $\begin{array}{l}\text { Skrypnika(a), } \\
2019[4]\end{array}$ & $\begin{array}{l}\text { Female } \\
(26 / 24)\end{array}$ & Poland & 56 & $\begin{array}{l}\text { probiotic mixture/ } \\
2.5 \times 10^{9}\end{array}$ & Placebo & 12 & $\begin{array}{l}\text { Obesity } \\
(\mathrm{BMl}>30 \\
\left.\mathrm{kg} / \mathrm{m}^{2}\right)\end{array}$ & $\begin{array}{l}\text { FFM\% was } \\
\text { measured by } \\
\text { BIA }\end{array}$ \\
\hline $\begin{array}{l}\text { Skrypnika(b), } \\
2019[4]\end{array}$ & $\begin{array}{l}\text { Female } \\
(23 / 24)\end{array}$ & Poland & 56 & $\begin{array}{l}\text { probiotic mixture/ } \\
1 \times 10^{10}\end{array}$ & Placebo & 12 & $\begin{array}{l}\text { Obesity } \\
(\mathrm{BMl}>30 \\
\left.\mathrm{kg} / \mathrm{m}^{2}\right)\end{array}$ & $\begin{array}{l}\text { FFM\% was } \\
\text { measured by } \\
\text { BIA }\end{array}$ \\
\hline $\begin{array}{l}\text { Szulinska(a), } \\
2018 \text { [5] }\end{array}$ & $\begin{array}{l}\text { Female } \\
(23 / 24)\end{array}$ & Poland & 56 & $\begin{array}{l}\text { probiotic mixture/ } \\
1 \times 10^{10}\end{array}$ & Placebo & 12 & $\begin{array}{l}\text { Obesity } \\
(\mathrm{BMI}=30-45 \\
\left.\mathrm{kg} / \mathrm{m}^{2}\right)\end{array}$ & $\begin{array}{l}\text { FFM\% was } \\
\text { measured by } \\
\text { BIA }\end{array}$ \\
\hline $\begin{array}{l}\text { Szulinska(b), } \\
2018 \text { [5] }\end{array}$ & $\begin{array}{l}\text { Female } \\
(24 / 24)\end{array}$ & Poland & 56 & $\begin{array}{l}\text { probiotic mixture/ } \\
2.5 \times 10^{9}\end{array}$ & Placebo & 12 & $\begin{array}{l}\text { obesity } \\
(\mathrm{BMl}=30- \\
\left.45 \mathrm{~kg} / \mathrm{m}^{2}\right)\end{array}$ & $\begin{array}{l}\text { FFM\% was } \\
\text { measured by } \\
\text { BIA }\end{array}$ \\
\hline Ford, 2020 [6] & $\begin{array}{l}\text { Female } \\
(7 / 6)\end{array}$ & USA & 73.7 & $\begin{array}{l}\text { HPD plus multi- } \\
\text { strain probiotic/ } \\
1.54 \times 10^{9}\end{array}$ & $\begin{array}{l}\text { Encapsulated } \\
\text { potato starch }\end{array}$ & 18 & Healthy & $\begin{array}{l}\text { FFM\% was } \\
\text { measured by } \\
\text { BIA }\end{array}$ \\
\hline \multicolumn{9}{|l|}{$\begin{array}{l}\text { Muscle } \\
\text { strength }\end{array}$} \\
\hline Lei, 2016 [7] & $\begin{array}{l}\text { Female/Male } \\
(189 / 192)\end{array}$ & China & $>60$ & $\begin{array}{l}\text { Lactobacillus/ } \\
6 \times 10^{9}\end{array}$ & Placebo & 24 & $\begin{array}{l}\text { non- } \\
\text { displaced } \\
\text { distal } \\
\text { radius } \\
\text { fracture }\end{array}$ & $\begin{array}{l}\text { Hand grip } \\
\text { strength }\end{array}$ \\
\hline Neto, 2013 [2] & $\begin{array}{l}\text { Female/Male } \\
(9 / 8)\end{array}$ & Brazil & 67.9 & $\begin{array}{l}\text { Frutooligossacarid- } \\
\text { probiotic mixture / } \\
1 \times 10^{9} \text { probiotic }\end{array}$ & Maltodextrin & 12 & Frailty & $\begin{array}{l}\text { Hand grip } \\
\text { strength }\end{array}$ \\
\hline Ford, 2020 [6] & $\begin{array}{l}\text { Female } \\
(21 / 23)\end{array}$ & USA & 73.7 & $\begin{array}{l}\text { HPD plus multi- } \\
\text { strain probiotic/ } \\
1.54 \times 10^{9}\end{array}$ & $\begin{array}{l}\text { Encapsulated } \\
\text { potato-starch }\end{array}$ & 18 & Healthy & $\begin{array}{l}\text { Hand grip } \\
\text { strength }\end{array}$ \\
\hline $\begin{array}{l}\text { Roman, } 2019 \\
\text { [8] }\end{array}$ & $\begin{array}{l}\text { Female/Male } \\
(17 / 18)\end{array}$ & Spain & 65.8 & $\begin{array}{l}\text { probiotic mixture/ } \\
450 \text { billion live } \\
\text { bacteria } \\
\text { per } 4.4 \mathrm{~g} \text { sachet }\end{array}$ & $\begin{array}{l}\text { maltose and } \\
\text { silicon dioxide }\end{array}$ & 12 & $\begin{array}{l}\text { Cognitive } \\
\text { dysfunction }\end{array}$ & $\begin{array}{l}\text { Hand grip } \\
\text { strength }\end{array}$ \\
\hline \multicolumn{9}{|l|}{$\begin{array}{l}\text { Body mass } \\
\text { index }\end{array}$} \\
\hline $\begin{array}{l}\text { Inoue, } 2018 \\
\text { [1] }\end{array}$ & $\begin{array}{l}\text { Female/Male } \\
(20 / 18)\end{array}$ & Japan & 71 & $\begin{array}{l}\text { Bifidobacterium/ } \\
1.25 \times 10^{10}\end{array}$ & Water & 12 & Healthy & $\begin{array}{l}\text { Body mass } \\
\text { index }\left(\mathrm{kg} / \mathrm{m}^{2}\right)\end{array}$ \\
\hline $\begin{array}{l}\text { Hwang, } 2019 \\
{[9]}\end{array}$ & $\begin{array}{l}\text { Female/Male } \\
(45 / 47)\end{array}$ & $\begin{array}{l}\text { South } \\
\text { Korea }\end{array}$ & 68.0 & $\begin{array}{l}\text { Lactobacillus } \\
\text { plantarum } \text { C29/1.25 } \\
\times 10^{10}\end{array}$ & cellulose & 12 & $\begin{array}{l}\text { Mild } \\
\text { Cognitive } \\
\text { Impairment }\end{array}$ & $\begin{array}{l}\text { Body mass } \\
\text { index }\left(\mathrm{kg} / \mathrm{m}^{2}\right)\end{array}$ \\
\hline
\end{tabular}




\begin{tabular}{|c|c|c|c|c|c|c|c|c|}
\hline Author, year & $\begin{array}{l}\text { Sex (F/M), subjects } \\
\text { (Intervention/placebo) }\end{array}$ & Country & $\begin{array}{l}\text { Age } \\
\text { (year) }\end{array}$ & $\begin{array}{l}\text { Type of Intervention } \\
\text { and dosage } \\
\text { (CFU/day) }\end{array}$ & $\begin{array}{l}\text { Type of } \\
\text { placebo }\end{array}$ & $\begin{array}{l}\text { Study } \\
\text { Duration } \\
\text { (weeks) }\end{array}$ & $\begin{array}{l}\text { Health } \\
\text { status of } \\
\text { subjects }\end{array}$ & Measurements \\
\hline $\begin{array}{l}\text { Mañé(a), } \\
2011[10]\end{array}$ & $\begin{array}{l}\text { Female/Male } \\
(19 / 18)\end{array}$ & Spain & 71 & $\begin{array}{l}\text { Probiotic mixture/ } \\
5.10^{10}\end{array}$ & placebo & 12 & Healthy & $\begin{array}{l}\text { Body mass } \\
\text { index }\left(\mathrm{kg} / \mathrm{m}^{2}\right)\end{array}$ \\
\hline $\begin{array}{l}\text { Mañé(b), } \\
2011 \text { [10] }\end{array}$ & $\begin{array}{l}\text { Female/Male } \\
(13 / 18)\end{array}$ & Spain & 70 & $\begin{array}{l}\text { Probiotic mixture/ } \\
5.10^{8}\end{array}$ & placebo & 12 & Healthy & $\begin{array}{l}\text { Body mass } \\
\text { index }\left(\mathrm{kg} / \mathrm{m}^{2}\right)\end{array}$ \\
\hline $\begin{array}{l}\text { Sato, } 2017 \\
\text { [11] }\end{array}$ & $\begin{array}{l}\text { Female/Male } \\
(34 / 34)\end{array}$ & Japan & 65 & $\begin{array}{l}\text { Lactobacillus- } \\
\text { fermented milk/ } \\
4 \times 10^{10}\end{array}$ & placebo & 16 & $\begin{array}{l}\text { Type } 2 \\
\text { diabetes }\end{array}$ & $\begin{array}{l}\text { Body mass } \\
\text { index }\left(\mathrm{kg} / \mathrm{m}^{2}\right)\end{array}$ \\
\hline $\begin{array}{l}\text { Shinkai(a), } \\
2012[12]\end{array}$ & $\begin{array}{l}\text { Female/Male } \\
(92 / 93)\end{array}$ & Japan & $70 \cdot 9$ & $\begin{array}{l}\text { Lactobacillus- } \\
\text { pentosus strain } \\
\text { b240/ } 2 \times 10^{9}\end{array}$ & placebo & 20 & Healthy & $\begin{array}{l}\text { Body mass } \\
\text { index }\left(\mathrm{kg} / \mathrm{m}^{2}\right)\end{array}$ \\
\hline $\begin{array}{l}\text { Shinkai(b), } \\
2012[12]\end{array}$ & $\begin{array}{l}\text { Female/Male } \\
(93 / 93)\end{array}$ & Japan & $70 \cdot 9$ & $\begin{array}{l}\text { Lactobacillus } \\
\text { pentosus strain } \\
\text { b240/ } 2 \times 10^{10}\end{array}$ & placebo & 20 & Healthy & $\begin{array}{l}\text { Body mass } \\
\text { index }\left(\mathrm{kg} / \mathrm{m}^{2}\right)\end{array}$ \\
\hline $\begin{array}{l}\text { Szulińska(a), } \\
\text { (2018) [5] }\end{array}$ & $\begin{array}{l}\text { Female } \\
(23 / 24)\end{array}$ & Poland & 56 & $\begin{array}{l}\text { probiotic mixture/ } \\
1 \times 10^{10}\end{array}$ & placebo & 12 & Obesity & $\begin{array}{l}\text { Body mass } \\
\text { index }\left(\mathrm{kg} / \mathrm{m}^{2}\right)\end{array}$ \\
\hline $\begin{array}{l}\text { Szulińska(b), } \\
2018[5]\end{array}$ & $\begin{array}{l}\text { Female } \\
(24 / 24)\end{array}$ & Poland & 56 & $\begin{array}{l}\text { probiotic mixture/ } \\
2.5 \times 10^{9}\end{array}$ & placebo & 12 & Obesity & $\begin{array}{l}\text { Body mass } \\
\text { index }\left(\mathrm{kg} / \mathrm{m}^{2}\right)\end{array}$ \\
\hline $\begin{array}{l}\text { Lopes, } 2019 \\
\text { [13] }\end{array}$ & $\begin{array}{l}\text { Female/Male } \\
(29 / 29)\end{array}$ & Brazil & 63.1 & $\begin{array}{l}\text { probiotic dairy } \\
\text { drink } / 7.4 \times 10^{8} \pm \\
5.4 \times 10^{8} \\
\text { CFU } / 100 \mathrm{~mL}\end{array}$ & milk & 7 & CKD & $\begin{array}{l}\text { Body mass } \\
\text { index }\left(\mathrm{kg} / \mathrm{m}^{2}\right)\end{array}$ \\
\hline $\begin{array}{l}\text { Yamamoto, } \\
2019[14]\end{array}$ & $\begin{array}{l}\text { Female/Male } \\
(44 / 52)\end{array}$ & Japan & 88.3 & $\begin{array}{l}\text { Yogurt enriched } \\
\text { with mixed } \\
\text { probiotics/ } 1.8 \text { and } \\
3.5 \times 10^{8}\end{array}$ & yogurt & 12 & Healthy & $\begin{array}{l}\text { Body mass } \\
\text { index }\left(\mathrm{kg} / \mathrm{m}^{2}\right)\end{array}$ \\
\hline Neto, 2013 [2] & $\begin{array}{l}\text { Female/Male } \\
(9 / 8)\end{array}$ & Brazil & 67.9 & $\begin{array}{l}\text { Frutooligossacari } \\
\text { probiotic mixture/ } \\
1 \times 10^{9} \text { probiotic }\end{array}$ & Maltodextrin & 12 & Frailty & $\begin{array}{l}\text { Body mass } \\
\text { index }\left(\mathrm{kg} / \mathrm{m}^{2}\right)\end{array}$ \\
\hline $\begin{array}{l}\text { Tamtaji, } 2019 \\
\text { [15] }\end{array}$ & $\begin{array}{l}\text { Female/Male } \\
(27 / 26)\end{array}$ & Iran & 76.2 & $\begin{array}{l}\text { Mixed probiotic / } \\
2 \times 10^{9}\end{array}$ & starch & 12 & $\begin{array}{l}\text { Alzheimer's } \\
\text { disease }\end{array}$ & $\begin{array}{l}\text { Body mass } \\
\text { index }\left(\mathrm{kg} / \mathrm{m}^{2}\right)\end{array}$ \\
\hline $\begin{array}{l}\text { Borzabadi, } \\
2018 \text { [16] }\end{array}$ & $\begin{array}{l}\text { Female/Male } \\
(25 / 25)\end{array}$ & Iran & 66.8 & $\begin{array}{l}\text { probiotic mixture/ } \\
8 \times 10^{9}\end{array}$ & Placebo & 12 & $\begin{array}{l}\text { Parkinson's } \\
\text { disease }\end{array}$ & $\begin{array}{l}\text { Body mass } \\
\text { index }\left(\mathrm{kg} / \mathrm{m}^{2}\right)\end{array}$ \\
\hline \multicolumn{9}{|l|}{ Body weight } \\
\hline $\begin{array}{l}\text { Hwang, } 2019 \\
\text { [9] }\end{array}$ & $\begin{array}{l}\text { Female/Male } \\
(45 / 47)\end{array}$ & $\begin{array}{l}\text { South } \\
\text { Korea }\end{array}$ & 68.0 & $\begin{array}{l}\text { Lactobacillus } \\
\text { plantarum } \text { C29/1.25 } \\
\times 10^{10}\end{array}$ & cellulose & 12 & $\begin{array}{l}\text { Mild } \\
\text { Cognitive } \\
\text { Impairment }\end{array}$ & $\begin{array}{l}\text { Body weight } \\
(\mathrm{kg})\end{array}$ \\
\hline $\begin{array}{l}\text { Yamamoto, } \\
2019[14]\end{array}$ & $\begin{array}{l}\text { Female/Male } \\
(44 / 52)\end{array}$ & Japan & 88.3 & $\begin{array}{l}\text { Yogurt enriched } \\
\text { with mixed } \\
\text { probiotic/ } 1.8 \text { and } \\
3.5 \times 10^{8}\end{array}$ & yogurt & 12 & Healthy & $\begin{array}{l}\text { Body weight } \\
(\mathrm{kg})\end{array}$ \\
\hline $\begin{array}{l}\text { Tamtaji, } 2019 \\
\text { [15] }\end{array}$ & $\begin{array}{l}\text { Female/Male } \\
(27 / 26)\end{array}$ & Iran & 76.2 & $\begin{array}{l}\text { probiotic mixture/ } 2 \\
\times 10^{9}\end{array}$ & starch & 12 & $\begin{array}{l}\text { Alzheimer's } \\
\text { disease }\end{array}$ & $\begin{array}{l}\text { Body weight } \\
(\mathrm{kg})\end{array}$ \\
\hline $\begin{array}{l}\text { Borzabadi, } \\
2018 \text { [16] }\end{array}$ & $\begin{array}{l}\text { Female/Male } \\
(25 / 25)\end{array}$ & Iran & 66.8 & $\begin{array}{l}\text { probiotic mixture/ } \\
8 \times 10^{9}\end{array}$ & Placebo & 12 & $\begin{array}{l}\text { Parkinson's } \\
\text { disease }\end{array}$ & $\begin{array}{l}\text { Body weight } \\
(\mathrm{kg})\end{array}$ \\
\hline $\begin{array}{l}\text { Macfarlane, } \\
2013 \text { [17] }\end{array}$ & $\begin{array}{l}\text { Female/Male } \\
(23 / 20)\end{array}$ & UK & 71.9 & $\begin{array}{l}\text { B. longum/ } \\
2 \times 10^{11}\end{array}$ & $\begin{array}{l}\text { potato and } \\
\text { maltodextrose }\end{array}$ & 4 & Healthy & Weight(kg) \\
\hline
\end{tabular}




\begin{tabular}{|c|c|c|c|c|c|c|c|c|}
\hline Author, year & $\begin{array}{l}\text { Sex (F/M), subjects } \\
\text { (Intervention/placebo) }\end{array}$ & Country & $\begin{array}{l}\text { Age } \\
\text { (year) }\end{array}$ & $\begin{array}{l}\text { Type of Intervention } \\
\text { and dosage } \\
\text { (CFU/day) }\end{array}$ & $\begin{array}{l}\text { Type of } \\
\text { placebo }\end{array}$ & $\begin{array}{l}\text { Study } \\
\text { Duration } \\
\text { (weeks) }\end{array}$ & $\begin{array}{l}\text { Health } \\
\text { status of } \\
\text { subjects }\end{array}$ & Measurements \\
\hline \multicolumn{9}{|l|}{ Body mass } \\
\hline $\begin{array}{l}\text { Inoue, } 2018 \\
\text { [1] }\end{array}$ & $\begin{array}{l}\text { Female/Male } \\
(20 / 18)\end{array}$ & Japan & 71 & $\begin{array}{l}\text { Bifidobacterium/ } \\
1.25 \times 10^{10}\end{array}$ & Water & 12 & Healthy & $\begin{array}{l}\text { Body mass } \\
(\mathrm{Kg})\end{array}$ \\
\hline $\begin{array}{l}\text { Skrypnik (a), } \\
2019[4]\end{array}$ & $\begin{array}{l}\text { Female } \\
(23 / 24)\end{array}$ & Poland & 56 & $\begin{array}{l}\text { probiotic mixture/ } \\
1 \times 10^{10}\end{array}$ & placebo & 12 & Obesity & $\begin{array}{l}\text { Body mass } \\
(\mathrm{Kg})\end{array}$ \\
\hline $\begin{array}{l}\text { Skrypnik (b), } \\
2019 \text { [4] }\end{array}$ & $\begin{array}{l}\text { Female } \\
(26 / 24)\end{array}$ & Poland & 56 & $\begin{array}{l}\text { probiotic mixture/ } \\
2.5 \times 10^{9}\end{array}$ & placebo & 12 & Obesity & $\begin{array}{l}\text { Body mass } \\
(\mathrm{Kg})\end{array}$ \\
\hline $\begin{array}{l}\text { Szulińska(a), } \\
2018 \text { [5] }\end{array}$ & $\begin{array}{l}\text { Female } \\
(23 / 24)\end{array}$ & Poland & 56 & $\begin{array}{l}\text { probiotic mixture/ } \\
1 \times 10^{10}\end{array}$ & placebo & 12 & Obesity & $\begin{array}{l}\text { Body mass } \\
(\mathrm{Kg})\end{array}$ \\
\hline $\begin{array}{l}\text { Szulińska(b), } \\
2018 \text { [5] }\end{array}$ & $\begin{array}{l}\text { Female } \\
(24 / 24)\end{array}$ & Poland & 56 & $\begin{array}{l}\text { probiotic mixture/ } \\
2.5 \times 10^{9}\end{array}$ & placebo & 12 & Obesity & $\begin{array}{l}\text { Body mass } \\
(\mathrm{Kg})\end{array}$ \\
\hline Neto, 2013 [2] & $\begin{array}{l}\text { Female/Male } \\
(9 / 8)\end{array}$ & Brazil & 67.9 & $\begin{array}{l}\text { probiotic mixture } \\
\text { with Frutooligos / } \\
1 \times 10^{9} \text { probiotic }\end{array}$ & Maltodextrin & 12 & Frailty & $\begin{array}{l}\text { Body mass } \\
(\mathrm{Kg})\end{array}$ \\
\hline $\begin{array}{l}\text { Waist } \\
\text { circumference }\end{array}$ & & & & & & & & \\
\hline $\begin{array}{l}\text { Szulińska(a), } \\
\text { (2018) [5] }\end{array}$ & $\begin{array}{l}\text { Female } \\
(23 / 24)\end{array}$ & Poland & 56 & $\begin{array}{l}\text { probiotic mixture/ } \\
1 \times 10^{10}\end{array}$ & placebo & 12 & Obesity & $\begin{array}{l}\text { Waist } \\
\text { circumference } \\
\text { (cm) }\end{array}$ \\
\hline $\begin{array}{l}\text { Szulińska(b), } \\
\text { (2018) [5] }\end{array}$ & $\begin{array}{l}\text { Female } \\
(24 / 24)\end{array}$ & Poland & 56 & $\begin{array}{l}\text { probiotic mixture/ } \\
2.5 \times 10^{9}\end{array}$ & placebo & 12 & Obesity & $\begin{array}{l}\text { Waist } \\
\text { circumference } \\
\text { (cm) }\end{array}$ \\
\hline $\begin{array}{l}\text { Waist to hip } \\
\text { ratio }\end{array}$ & & & & & & & & \\
\hline $\begin{array}{l}\text { Hlivak, } 2005 \\
\text { [18] }\end{array}$ & $\begin{array}{l}\text { Female/Male } \\
(20 / 18)\end{array}$ & Slovakia & 75.35 & $\begin{array}{l}\text { probiotic mixture } \\
(E F M-74) / 2 \times 10^{9}\end{array}$ & placebo & 12 & Healthy & $\begin{array}{l}\text { Waist to hip } \\
\text { ratio }\end{array}$ \\
\hline $\begin{array}{l}\text { F: Female; M: } \\
\text { Centimeters; } \mathrm{C}\end{array}$ & $\begin{array}{l}\text { le; BIA: Bioelectrical imp } \\
\text { : Chronic kidney disease }\end{array}$ & $\begin{array}{l}\text { dance anal } \\
\text { BMD: bone }\end{array}$ & $\begin{array}{l}\text { sis; Kg: } \\
\text { mineral }\end{array}$ & $\begin{array}{l}\text { ilogram; FFM: Fat Fre€ } \\
\text { ensity; CFU: Colony Fo }\end{array}$ & $\begin{array}{l}\text { Mass; BMI: Bod } \\
\text { ning Units }\end{array}$ & Mass Index; & D: Standa & viation; cm: \\
\hline $\begin{array}{l}\text { 1. Inoue, T., et } \\
\text { habits of healt }\end{array}$ & $\begin{array}{l}\text { Effect of combined bific } \\
\text { elderly subjects. Benef }\end{array}$ & $\begin{array}{l}\text { bacteria } s \\
\text { icrobes, } 20\end{array}$ & $\begin{array}{l}\text { pplemer } \\
\text { 8. } 9(6):\end{array}$ & $\begin{array}{l}\text { ation and resistance tr } \\
843-853 \text {. }\end{array}$ & ining on cognit & function, $b c$ & dy compos & and bowel \\
\hline $\begin{array}{l}\text { 2. Neto, J.V., C. } \\
\text { pilot study. Nut }\end{array}$ & $\begin{array}{l}\text { De Melo, and S.M.L. Rib } \\
\text { ents, 2013. 5(4): p. 1276- }\end{array}$ & $\begin{array}{l}\text { iro, Effects } \\
286 .\end{array}$ & of three- & nonth intake of synbio & con inflammat & and body c & omposition & he elderly: a \\
\hline $\begin{array}{l}\text { 3. Nilsson, A.G } \\
\text { double-blind, } c\end{array}$ & $\begin{array}{l}\text { t al., Lactobacillus reute } \\
\text { hical trial. J Intern Med, } 2\end{array}$ & $\begin{array}{l}\text { reduces bc } \\
\text { 18. 284(3): }\end{array}$ & $\begin{array}{l}\text { ne loss i } \\
\text { b. } 307-31\end{array}$ & $\begin{array}{l}\text { older women with low } \\
7 .\end{array}$ & one mineral de & ity: a randor & nized, place & controlled, \\
\hline $\begin{array}{l}\text { 4. Skrypnik, K., } \\
\text { randomized tri }\end{array}$ & $\begin{array}{l}\text { al., The effect of multist } \\
\text { Food Funct, 2019. 10(8) }\end{array}$ & $\begin{array}{l}\text { ain probiot } \\
\text { p. } 5228-52\end{array}$ & $\begin{array}{l}\text { supple } \\
8 .\end{array}$ & nentation in two doses & in iron metaboli & in obese $p$ & stmenopa & women: a \\
\hline $\begin{array}{l}\text { 5. Szulińska, N } \\
\text { Cardiometabol }\end{array}$ & $\begin{array}{l}\text { et al., Dose-Dependent Et } \\
\text { Profile in Obese Postme }\end{array}$ & $\begin{array}{l}\text { ects of Mu } \\
\text { opausal W }\end{array}$ & $\begin{array}{l}\text { ispecies } \\
\text { men: } A\end{array}$ & $\begin{array}{l}\text { Probiotic Supplementa } \\
\text { 2-Week Randomized C }\end{array}$ & $\begin{array}{l}\text { on on the Lipop } \\
\text { nical Trial. Nutri }\end{array}$ & $\begin{array}{l}\text { ysaccharide } \\
\text { ts, } 2018.10\end{array}$ & $\begin{array}{l}\text { (LPS) Leve } \\
6) \text {. }\end{array}$ & \\
\hline $\begin{array}{l}\text { 6. Ford, A.L., et } \\
\text { synbiotic: } A \text { ral } \\
\text { 500-516. e10. }\end{array}$ & $\begin{array}{l}\text { I., Microbiota stability an } \\
\text { omized, double-blind, pla }\end{array}$ & $\begin{array}{l}\text { gastrointe } \\
\text { cebo-contrc }\end{array}$ & $\begin{array}{l}\text { tinal tole } \\
\text { led trial }\end{array}$ & $\begin{array}{l}\text { ance in response to a } \\
\text { older women. Journa }\end{array}$ & $\begin{array}{l}\text { igh-protein diet } \\
\text { of the Academy }\end{array}$ & $\begin{array}{l}\text { th and witho } \\
\text { Nutrition a }\end{array}$ & $\begin{array}{l}\text { ut a prebio } \\
\text { d Dietetics }\end{array}$ & $\begin{array}{l}\text { robiotic, and } \\
\text { 20. } 120(4): \text { p. }\end{array}$ \\
\hline $\begin{array}{l}\text { 7. Lei, M., L. Hu } \\
\text { controlled ranc }\end{array}$ & $\begin{array}{l}\text { and D. Wang, The effect } \\
\text { mised clinical trial. Benef }\end{array}$ & $\begin{array}{l}\text { of probiotic } \\
\text { cial microb }\end{array}$ & $\begin{array}{l}\text { treatme } \\
\mathrm{s}, 2016 .\end{array}$ & $\begin{array}{l}\text { ton elderly patients wi } \\
\text { (5): p. 631-637. }\end{array}$ & distal radius $f$ & ture: a pros & ective dou & lind, placebo- \\
\hline $\begin{array}{l}\text { 8. Román, E., e } \\
\text { communicatio }\end{array}$ & $\begin{array}{l}\text { al., Effect of a multistrain } \\
\text {, 2019. 3(5): p. 632-645. }\end{array}$ & probiotic or & cognitiv & function and risk of $f_{c}$ & Is in patients w & cirrhosis: $A$ & randomize & I. Hepatology \\
\hline $\begin{array}{l}\text { 9. Hwang, Y.-H. } \\
\text { impairment: a }\end{array}$ & $\begin{array}{l}\text { et al., Efficacy and safety } \\
\text { 2-week, multi-center, rand }\end{array}$ & $\begin{array}{l}\text { f Lactobac } \\
\text { mized, dou }\end{array}$ & $\begin{array}{l}\text { Illus plar } \\
\text { le-blind, }\end{array}$ & $\begin{array}{l}\text { arum C29-fermented s } \\
\text { lacebo-controlled clin }\end{array}$ & $\begin{array}{l}\text { ybean (DW200 } \\
\text { al trial. Nutrient }\end{array}$ & $\begin{array}{l}\text { in individual } \\
\text { 2019. 11(2): }\end{array}$ & $\begin{array}{l}\text { with mild } \\
\text { p. } 305 \text {. }\end{array}$ & nitive \\
\hline $\begin{array}{l}\text { 10. Mañé, J., e } \\
\text { response, douk }\end{array}$ & $\begin{array}{l}\text { A mixture of Lactobac } \\
\text { blind, placebo-controlle }\end{array}$ & $\begin{array}{l}\text { lus planta } \\
\text { randomiz }\end{array}$ & $\begin{array}{l}n \text { CECT } \\
\text { pilot tr }\end{array}$ & $\begin{array}{l}315 \text { and CECT } 7316 \\
\text {. Nutr Hosp, 2011. } 26\end{array}$ & $\begin{array}{l}\text { hances system } \\
\text { : p. 228-35. }\end{array}$ & mmunity i & elderly sut & A dose- \\
\hline
\end{tabular}




\begin{tabular}{|c|c|c|c|c|c|c|c|c|}
\hline Author, year & $\begin{array}{l}\text { Sex (F/M), subjects } \\
\text { (Intervention/placebo) }\end{array}$ & Country & $\begin{array}{l}\text { Age } \\
\text { (year) }\end{array}$ & $\begin{array}{l}\text { Type of Intervention } \\
\text { and dosage } \\
\text { (CFU/day) }\end{array}$ & $\begin{array}{l}\text { Type of } \\
\text { placebo }\end{array}$ & $\begin{array}{l}\text { Study } \\
\text { Duration } \\
\text { (weeks) }\end{array}$ & $\begin{array}{l}\text { Health } \\
\text { status of } \\
\text { subjects }\end{array}$ & Measurements \\
\hline
\end{tabular}

11. Sato, J., et al., Probiotic reduces bacterial translocation in type 2 diabetes mellitus: A randomised controlled study. Scientific reports, 2017. 7(1): p. $1-10$.

12. Shinkai, S., et al., Immunoprotective effects of oral intake of heat-killed Lactobacillus pentosus strain b240 in elderly adults: a randomised, double-blind, placebo-controlled trial. Br J Nutr, 2013. 109(10): p. 1856-65.

13. Lopes, R.d.C.S.O., et al., Evaluation of the health benefits of consumption of extruded tannin sorghum with unfermented probiotic milk in individuals with chronic kidney disease. Food Research International, 2018. 107: p. 629-638.

14. Yamamoto, Y., et al., Effect of ingesting yogurt fermented with Lactobacillus delbrueckii ssp. bulgaricus OLL1073R-1 on influenza virus-bound salivary IgA in elderly residents of nursing homes: a randomized controlled trial. Acta Odontol Scand, 2019. 77(7): p. 517-524.

15. Tamtaji, O.R., et al., Probiotic and selenium co-supplementation, and the effects on clinical, metabolic and genetic status in Alzheimer's disease: A randomized, double-blind, controlled trial. Clin Nutr, 2019. 38(6): p. 2569-2575.

16. Borzabadi, S., et al., The effects of probiotic supplementation on gene expression related to inflammation, insulin and lipid in patients with Parkinsonâ s disease: A randomized, double-blind, placebo-controlled trial. Archives of Iranian medicine, 2018. 21(7): p. $289-295$.

17. Macfarlane, S., et al., Synbiotic consumption changes the metabolism and composition of the gut microbiota in older people and modifies inflammatory processes: a randomised, double-blind, placebo-controlled crossover study. Aliment Pharmacol Ther, 2013. 38(7): p. 804-16.

18. Hlivak, P., et al., One-year application of probiotic strain Enterococcus faecium M-74 decreases serum cholesterol levels. Bratisl Lek Listy, 2005. 106(2): p. 67-72.

\subsection{Risk of bias assessment}

Based on the criteria of applied tool designed for randomized clinical trials (Cochrane quality assessment tool) (Figure 2), 6 studies had a low risk of bias $[22,23,28,29,32,37]$. In 9 studies, the overall risk of bias was high [20, 21, 24, 25, 30, 31, 34-36]. Among included studies, the conducted studies by Valentini Neto et al. and Ford et al. had a moderate risk of bias in the current systematic review [27].

\subsection{The impact of probiotic supplements on fat free mass and muscle mass}

The overall effect of meta-analysis on seven studies showed a null effect of probiotic supplementation on muscle mass (WMD: -0.05, 95\% Cl: -1.54, 1.43; I-square: $0.0 \%, P=0.985)$. Sub-group analysis based on muscle mass (Kg) and fat-free mass (\%) also showed no significant effect of probiotic supplementation on aforementioned measurements (WMD: 0.07, 95\% Cl: $-1.78,1.92$; l-square: $0.0 \%, P=0.644)$ for muscle mass (Kg) and (WMD: -0.27 , 95\% Cl: $-2.76,2.21$; I-square: 0.0\%, P=0.993) for fat-free mass (\%) (Figure 3).

\subsection{The impact of probiotic supplements on muscle strength}

Based on the pooled overall effect of five studies with totally 505 participants, probiotic supplementation had a non-significant effect on muscle strength (WMD: 0.7, 95\% Cl: -0.01, 1.40; I-square: 76.8\%, P=0.005) (Figure 4). To reduce the substantial heterogeneity, a sub-group analysis was run based on the duration of interventions. The result of the analysis showed probiotic supplementation more than 12 weeks significantly increased muscle strength (WMD: $1.15,95 \% \mathrm{Cl}: 0.86,1.43$; I-square: $0.0 \%, \mathrm{P}=0.679$ ). However, probiotic supplementation less than 12 weeks had no effect on muscle strength (WMD: $-0.48,95 \% \mathrm{Cl}:-1.35,0.39$; I-square: 0.0\%, P=0.413) (Figure 4).

\subsection{The impact of probiotic supplements on anthropometric measurements}

The results of meta-analysis on anthropometric measurements including body mass index, and body mas are presented in Table 2 . Accordingly, probiotic supplementation had no significant effect on anthropometric indices, including body mass and body mass index. In addition, there was no substantial heterogeneity between studies in all analyses.

Table 2

Effects of probiotic supplementation on anthropometric measurements

\begin{tabular}{|lllll|}
\hline & No. of studies & $\operatorname{SMD}(95 \% \mathrm{Cl})$ & $\mathrm{I}^{2}(\%)$ & Pvalue for Heterogeneity \\
\hline Body mass index & 12 & $0.08(-0.16,0.32)$ & 0.0 & 0.718 \\
\hline Body mass & 6 & $-0.05(-2.57,1.56)$ & 0.0 & 0.976 \\
\hline
\end{tabular}

\subsection{Sensitivity analysis}

The sensitivity analysis regarding the effect of probiotic supplementation on muscle strength specified that, after excluding an unmatched study[29], heterogeneity between studies was disappeared (WMD: $1.4[0.86,1.42])$. However, concerning impact of probiotic supplementation on muscle mass, body mass, $\mathrm{BMI}$, and body weight, there were no evidences of publication bias for included studies.

\section{Discussion}


The results of this systematic review and meta-analysis point out a null effect of probiotic supplementation on muscle mass, muscle strength, and anthropometric measurements. However, sub-group analysis affirmed that probiotic supplementation for more than 12 weeks can increase muscle strength significantly.

Sarcopenia is a worldwide health problem with adverse outcomes that needs immediate medical and nutritional interventions [39]. In current study, we showed that, after sub-group analysis, supplementation with probiotic for more than 12 weeks can improve muscle strength. Also we indicated that the excluding an unmatched study could disclose the positive effect of probiotic administration on muscle strength beyond the duration of supplementation. Recently, the important role of gut microbiota in inducing the cycle of age-related muscle dysfunction has been suggested [8, 40]. Consistent with our research, animal studies showed that probiotic/prebiotic administration as dietary supplement could ameliorate age-related muscle dysfunctions through the gut-muscle axis[40,41]. A recent observational study by Bjørkhaugh et al. also showed that the alterations of fecal microbiota composition by alcohol overconsumption had a significant inverse correlation with hand-grip strength, and was associated with the higher proinflammatory microenvironment[42]. It's believed that muscle dysfunctions and abnormal gut microbiota dysbiosis, in particular increased enterobacteria numbers, can occur simultaneously as consequences of aging [43]. Moreover, it's showed that aging and inflammation are closely related to each other, and higher levels of inflammatory markers are associated with sarcopenia, frailty, as well as morbidity and mortality among older persons[44]. According the previous literature, underlying causes of elevated inflammation are varied, among them changes in gut microbiota has a crucial role $[8,44]$. A recent meta-analysis on observational studies has shown that higher levels of inflammatory markers, including $C$ reactive protein (CRP), Interleukin-6 (IL-6), and Tumor necrosis factor- a (TNF-a) levels were related to muscle strength reduction in adults over time [45]. Our recent metaanalysis also indicated that increasing circulating levels of two pro-inflammatory markers, CRP and hs-CRP, were correlated with impairment of muscle strength among both the community population and patients. [46]. So, it is supposed that higher inflammation is an important risk factor for muscle atrophy and malfunction through interfering with muscle anabolism and energy homeostasis [47]. Interestingly, results of a previous systematic review and meta-analysis confirmed that probiotic supplementation has beneficial effects on circulating inflammatory biomarkers in health and disease conditions [48].

Reduced levels of IGF-1 (Insulin-like growth factor 1) simultaneous during life time may be another explanation for the potential association between probiotics and sarcopenia[49]. In this regard, lower handgrip strength and worse physical performance are documented in elderly persons with lower levels of IGF-1 levels $[50,51]$. Surprisingly, the predictive role of IGF-1 levels on muscle function is only observed in subjects with the lowest levels of inflammation (IL-6 levels); explaining the mediatory role of inflammation between IGF-1 and muscle strength and power [49]. It is previously well indicated that low expression of IGF-1 is correlated with impaired differentiation of myotubes resulting reduced size and dysfunction of skeletal muscles [51]. Moreover, animal studies suggested that Bifidobacterium infantis administration may up-regulate IGF-1 expression subsequent of lipopolysaccharide injection [52]. Given the mentioned reasons, the gut-muscle axis might clarify how probiotic supplementation can affect gut microorganism composition and increase overall health resulting from the improvement of inflammation and immune system function in older adults [53].

In addition to the improvement in muscle strength, some preceding research proposed the possible favorable effect of probiotic on muscle mass[54]. Unexpectedly, our analysis on seven clinical studies indicated the null effect of probiotic supplementation on muscle mass and anthropometric indices. Previous investigations in animal models demonstrated that alteration of gut microbiota can positively affect the skeletal muscle mass and function[55]. Also, the administration of probiotics in mouse models with muscle disorder improved muscle mass[40, 56]. In this concern, observational studies indicated that the reduction of gut microbiota biodiversity induced by aging is associated with loss of skeletal muscle and calf circumference reduction [57]. Additionally, the association between body mass index (BMI) and microbiota composition has been reported in Ukrainian obese persons[58]. Although the possible modulatory impact of probiotics on muscle mass deposition is theoretically proposed, however, due to the nature of observational studies, the relevant causation in this phenomenon has not been verified. Another possible reasons may be related to the complex etiology of age-related decline in muscle mass. Since, age-related muscle loss can be influenced by a variety of different factors rather than abnormal gut microbiota, including dietary protein intake and less physical activity[59]. It also should be considered that, among seven included studies in our polled analysis, participants of four studies were woman that may vitiate the overall results.[28, 32, 33, 38]. So, due to lack of consensus in this context, the appropriate intervention on muscle mass and anthropometric indices like probiotic supplementation is suggested in both healthy and sarcopenic elderly to demonstrate the different dimensions of applied treatment more clearly [27, 41].

Clinically, based on the last definition provided by the European Working Group on age-related sarcopenia, the reliability of muscle strength in predicting sarcopenia and its adverse outcomes is even more than muscle mass [3]. The outcomes of present study emphasize the clinical prominence of probiotic supplementation on the most reliable diagnostic sarcopenia component, muscle strength. Moreover, our outcomes may make known new insight regarding the important role of gastrointestinal tract on muscle function and dysfunction, and the assessment of the intestinal microbiota diversity can be a good prognostic tool for impaired muscle function. Furthermore, appropriate subgroup analysis enabled us to elicit a remarkable association between probiotic supplementation and muscle strength. These findings have recommended that the application of probiotics could be also justified for the prevention of age-related muscle dysfunction. However, some limitations must be considered. First, as a consequence of data deficiencies, the estimation of the overall pooled effect of probiotic supplementation on muscle performance was not possible. Second, we included all studies with various comorbid from a range of metabolic dysfunction and inpatients population to obese subjects in our analysis that might influence the results. Since, the underlying mechanism of sarcopenia might be different in sarcopenic-obese participants rather than elderly ones. Nevertheless, the limited number of studies that have been done among obese subjects, consequently, we could not run subgroup analysis. In addition, the available health status diversity (healthy vs. ill) between included studies might disturb our final findings. Finally, due to lack of evidence, we could not perform analysis

Page $9 / 16$ 
on inflammatory markers as well as IGF-1 levels to reinforce the proposed mechanism in the present study. The identifying possible mechanism underlying the positive effect of probiotics administration on muscle strength elderly subjects may recommend the specific probiotic preparation for a specific population group. So, more clinical trials on sarcopenic older subjects with simultaneous assessment inflammation as well as other possible pathogenesis variables, including adipokines levels, oxidative stress markers, insulin resistance, should be taken into concern for [60] possible mechanism underlying this finding.

\section{Conclusion}

In conclusion, this study declared the positive impact of probiotic supplementation on the most reliable diagnostic sarcopenia component, muscle strength (based on the last definitions by EWGSOP), in particular, probiotic administration for more than 12 weeks. However, more clinical trials on sarcopenic older subjects with simultaneous assessment inflammation as well as other possible pathogenesis variables is recommended.

\section{Declarations}

\section{Acknowledgments: None.}

Conflict of interests: There were no competing interests among authors

Statement of Ethics: An ethics statement is not applicable because this study is based exclusively on published literature. This study protocol was reviewed and approved by Ethic committee of Isfahan University of Medical Science, IR.MUI.RESEARCH.REC.1399.324.

Funding Sources: This study was supported by Isfahan University of Medical science. The funders had no role in the study design, data collection, and analysis, decision to publish, or preparation of the manuscript.

Author Contributions: N.Sh-M and S.S-B designed the study. N.Sh-M, Z.H, and F.N performed abstract screening and data extraction. M.R, and S.S-B analyzed the data and drafted the manuscript. All authors revised and approved the final manuscript.

Data Availability Statement: All data generated or analyzed during this study are included in this article and its supplementary material files. Further enquiries can be directed to the corresponding author.

\section{References}

1. Partridge, L., J. Deelen, and P.E. Slagboom, Facing up to the global challenges of ageing. Nature, 2018. 561(7721): p. 45-56.

2. Dhillon, R.J. and S. Hasni, Pathogenesis and Management of Sarcopenia. Clin Geriatr Med, 2017. 33(1): p. 17-26.

3. Cruz-Jentoft, A.J., et al., Sarcopenia: revised European consensus on definition and diagnosis. Age Ageing, 2019. 48(1): p. 16-31.

4. Woo, J., Sarcopenia. Clin Geriatr Med, 2017. 33(3): p. 305-314.

5. Iolascon, G., et al., Pharmacological therapy of sarcopenia: past, present and future. Clinical Cases in Mineral \& Bone Metabolism, 2018. 15(3).

6. Lakshminarayanan, B., et al., Compositional dynamics of the human intestinal microbiota with aging: Implications for health. Journal of Nutrition, Health and Aging, 2014. 18(9): p. 773-786.

7. Tiihonen, K., A.C. Ouwehand, and N. Rautonen, Human intestinal microbiota and healthy ageing. Ageing Research Reviews, 2010. 9(2): p. 107-116.

8. Ticinesi, A., et al., Aging gut microbiota at the cross-road between nutrition, physical frailty, and sarcopenia: Is there a gut-muscle axis? Nutrients, 2017. 9(12).

9. Ticinesi, A., et al., Gut microbiota, muscle mass and function in aging: A focus on physical frailty and sarcopenia. Nutrients, 2019. 11(7).

10. Ni, Y., et al., Lactobacillus and Bifidobacterium Improves Physiological Function and Cognitive Ability in Aged Mice by the Regulation of Gut Microbiota. Mol Nutr Food Res, 2019. 63(22): p. e1900603.

11. Siddharth, J., et al., Aging and sarcopenia associate with specific interactions between gut microbes, serum biomarkers and host physiology in rats. Aging, 2017. 9(7): p. 1698-1720.

12. Chen, Y.M., et al., Lactobacillus plantarum TWK10 Supplementation Improves Exercise Performance and Increases Muscle Mass in Mice. Nutrients, 2016. 8(4): p. 205.

13. Buigues, C., et al., Effect of a Prebiotic Formulation on Frailty Syndrome: A Randomized, Double-Blind Clinical Trial. Int J Mol Sci, 2016. 17(6).

14. Aoyagi, Y., et al., Independent and Interactive Effects of Habitually Ingesting Fermented Milk Products Containing Lactobacillus casei Strain Shirota and of Engaging in Moderate Habitual Daily Physical Activity on the Intestinal Health of Older People. Frontiers in Microbiology, 2019. 10.

15. Picca, A., et al., Gut Microbial, Inflammatory and Metabolic Signatures in Older People with Physical Frailty and Sarcopenia: Results from the BIOSPHERE Study. Nutrients, 2019. 12(1).

16. Sakata, S., et al., Effects of administration of lactobacillus curvatus cp2998 on physical function in the older adults-A Placebo-controlled, Randomized, Double-blind, Parallel-group Study. Japanese Pharmacology and Therapeutics, 2019. 47(6): p. 937-947.

17. Moher, D., et al., PRISMA statement. Epidemiology, 2011. 22(1): p. 128; author reply 128. 
18. Higgins, J.P. and S.e. Green, The Cochrane Collaboration. Cochrane handbook for systematic reviews of interventions, 2011. 4(6).

19. Hozo, S.P., B. Djulbegovic, and I. Hozo, Estimating the mean and variance from the median, range, and the size of a sample. BMC medical research methodology, 2005. 5(1): p. 1-10.

20. Borzabadi, S., et al., The effects of probiotic supplementation on gene expression related to inflammation, insulin and lipid in patients with Parkinsonâ s disease: A randomized, double-blind, placebo-controlled trial. Archives of Iranian medicine, 2018. 21(7): p. $289-295$.

21. Hlivak, P., et al., One-year application of probiotic strain Enterococcus faecium M-74 decreases serum cholesterol levels. Bratisl Lek Listy, 2005. 106(2): p. 67-72.

22. Inoue, T., et al., Effect of combined bifidobacteria supplementation and resistance training on cognitive function, body composition and bowel habits of healthy elderly subjects. Benef Microbes, 2018. 9(6): p. 843-853.

23. Lei, M., L. Hua, and D. Wang, The effect of probiotic treatment on elderly patients with distal radius fracture: a prospective double-blind, placebocontrolled randomised clinical trial. Beneficial microbes, 2016. 7(5): p. 631-637.

24. Lopes, R.d.C.S.O., et al., Evaluation of the health benefits of consumption of extruded tannin sorghum with unfermented probiotic milk in individuals with chronic kidney disease. Food Research International, 2018. 107: p. 629-638.

25. Macfarlane, S., et al., Synbiotic consumption changes the metabolism and composition of the gut microbiota in older people and modifies inflammatory processes: a randomised, double-blind, placebo-controlled crossover study. Aliment Pharmacol Ther, 2013. 38(7): p. 804-16.

26. Mañé, J., et al., A mixture of Lactobacillus plantarum CECT 7315 and CECT 7316 enhances systemic immunity in elderly subjects. A dose-response, double-blind, placebo-controlled, randomized pilot trial. Nutr Hosp, 2011. 26(1): p. 228-35.

27. Neto, J.V., C.M. De Melo, and S.M.L. Ribeiro, Effects of three-month intake of synbiotic on inflammation and body composition in the elderly: a pilot study. Nutrients, 2013. 5(4): p. 1276-1286.

28. Nilsson, A.G., et al., Lactobacillus reuteri reduces bone loss in older women with low bone mineral density: a randomized, placebo-controlled, doubleblind, clinical trial. J Intern Med, 2018. 284(3): p. 307-317.

29. Román, E., et al., Effect of a multistrain probiotic on cognitive function and risk of falls in patients with cirrhosis: A randomized trial. Hepatology communications, 2019. 3(5): p. 632-645.

30. Sato, J., et al., Probiotic reduces bacterial translocation in type 2 diabetes mellitus: A randomised controlled study. Scientific reports, 2017. 7(1): p. $1-10$.

31. Shinkai, S., et al., Immunoprotective effects of oral intake of heat-killed Lactobacillus pentosus strain b240 in elderly adults: a randomised, doubleblind, placebo-controlled trial. Br J Nutr, 2013. 109(10): p. 1856-65.

32. Skrypnik, K., et al., The effect of multistrain probiotic supplementation in two doses on iron metabolism in obese postmenopausal women: a randomized trial. Food Funct, 2019. 10(8): p. 5228-5238.

33. Szulińska, M., et al., Dose-Dependent Effects of Multispecies Probiotic Supplementation on the Lipopolysaccharide (LPS) Level and Cardiometabolic Profile in Obese Postmenopausal Women: A 12-Week Randomized Clinical Trial. Nutrients, 2018. 10(6).

34. Tamtaji, O.R., et al., Probiotic and selenium co-supplementation, and the effects on clinical, metabolic and genetic status in Alzheimer's disease: $A$ randomized, double-blind, controlled trial. Clin Nutr, 2019. 38(6): p. 2569-2575.

35. Yamamoto, Y., et al., Effect of ingesting yogurt fermented with Lactobacillus delbrueckii ssp. bulgaricus OLL1073R-1 on influenza virus-bound salivary IgA in elderly residents of nursing homes: a randomized controlled trial. Acta Odontol Scand, 2019. 77(7): p. 517-524.

36. Hwang, Y.-H., et al., Efficacy and safety of Lactobacillus plantarum C29-fermented soybean (DW2009) in individuals with mild cognitive impairment: a 12-week, multi-center, randomized, double-blind, placebo-controlled clinical trial. Nutrients, 2019. 11(2): p. 305.

37. Ford, A.L., et al., Microbiota Stability and Gastrointestinal Tolerance in Response to a High-Protein Diet with and without a Prebiotic, Probiotic, and Synbiotic: A Randomized, Double-Blind, Placebo-Controlled Trial in Older Women. J Acad Nutr Diet, 2020. 120(4): p. 500-516.e10.

38. Ford, A.L., et al., Microbiota stability and gastrointestinal tolerance in response to a high-protein diet with and without a prebiotic, probiotic, and synbiotic: A randomized, double-blind, placebo-controlled trial in older women. Journal of the Academy of Nutrition and Dietetics, 2020. 120(4): p. 500-516. e10.

39. Beaudart, C., et al., Sarcopenia: burden and challenges for public health. Archives of Public Health, 2014. 72(1): p. 1-8.

40. Chen, L.H., et al., Probiotic supplementation attenuates age-related sarcopenia via the gut-muscle axis in SAMP8 mice. Journal of Cachexia, Sarcopenia and Muscle, 2021.

41. Liu, C., et al., Understanding the gut microbiota and sarcopenia: a systematic review. Journal of Cachexia, Sarcopenia and Muscle, 2021.

42. Bjørkhaug, S.T., et al., Characterization of gut microbiota composition and functions in patients with chronic alcohol overconsumption. Gut microbes, 2019. 10(6): p. 663-675.

43. Ni Lochlainn, M., R.C. Bowyer, and C.J. Steves, Dietary protein and muscle in aging people: the potential role of the gut microbiome. Nutrients, 2018. 10(7): p. 929.

44. Ferrucci, L. and E. Fabbri, Inflammageing: chronic inflammation in ageing, cardiovascular disease, and frailty. Nature Reviews Cardiology, 2018. 15(9): p. 505-522. 
45. Tuttle, C.S., L.A. Thang, and A.B. Maier, Markers of inflammation and their association with muscle strength and mass: $A$ systematic review and meta-analysis. Ageing research reviews, 2020: p. 101185.

46. Shokri-Mashhadi, N., et al., Association of circulating C-reactive protein and high-sensitivity C-reactive protein with components of sarcopenia: $A$ systematic review and meta-analysis of observational studies. Experimental gerontology, 2021: p. 111330.

47. Belizário, J.E., et al., Skeletal muscle wasting and renewal: a pivotal role of myokine IL-6. Springerplus, 2016. 5(1): p. 1-15.

48. Kazemi, A., et al., Effect of probiotic and synbiotic supplementation on inflammatory markers in health and disease status: $A$ systematic review and meta-analysis of clinical trials. Clinical Nutrition, 2020. 39(3): p. 789-819.

49. Barbieri, M., et al., Chronic inflammation and the effect of IGF-I on muscle strength and power in older persons. American Journal of PhysiologyEndocrinology and Metabolism, 2003. 284(3): p. E481-E487.

50. Van Nieuwpoort, I., et al., The relationship between serum IGF-1, handgrip strength, physical performance and falls in elderly men and women. European journal of endocrinology, 2018. 179(2): p. 73-84.

51. Hor, Y.Y., et al., The molecular mechanisms of probiotic strains in improving ageing bone and muscle of d-galactose-induced ageing rats. Journal of applied microbiology, 2021. 130(4): p. 1307-1322.

52. Wang, W., et al., Effects of Bifidobacterium infantis on cytokine-induced neutrophil chemoattractant and insulin-like growth factor-1 in the ileum of rats with endotoxin injury. World journal of gastroenterology, 2019. 25(23): p. 2924.

53. Sanchez, B., et al., Probiotics, gut microbiota, and their influence on host health and disease. Mol Nutr Food Res, 2017. 61(1).

54. Ticinesi, A., et al., Gut Microbiota, Muscle Mass and Function in Aging: A Focus on Physical Frailty and Sarcopenia. Nutrients, 2019. 11(7): p. 1633.

55. Lahiri, S., et al., The gut microbiota influences skeletal muscle mass and function in mice. Science translational medicine, 2019. 11(502).

56. Varian, B.J., et al., Beneficial bacteria inhibit cachexia. Oncotarget, 2016. 7(11): p. 11803.

57. Ren, G., et al., Gut microbiota composition influences outcomes of skeletal muscle nutritional intervention via blended protein supplementation in posttransplant patients with hematological malignancies. Clinical Nutrition, 2021. 40(1): p. 94-102.

58. Koliada, A., et al., Association between body mass index and Firmicutes/Bacteroidetes ratio in an adult Ukrainian population. BMC microbiology, 2017. 17(1): p. 120.

59. Martin-Cantero, A., et al., Factors influencing the efficacy of nutritional interventions on muscle mass in older adults: a systematic review and metaanalysis. Nutrition reviews, 2021. 79(3): p. 315-330.

60. Polyzos, S.A. and A.N. Margioris, Sarcopenic obesity. Hormones, 2018. 17(3): p. 321-331.

\section{Figures}




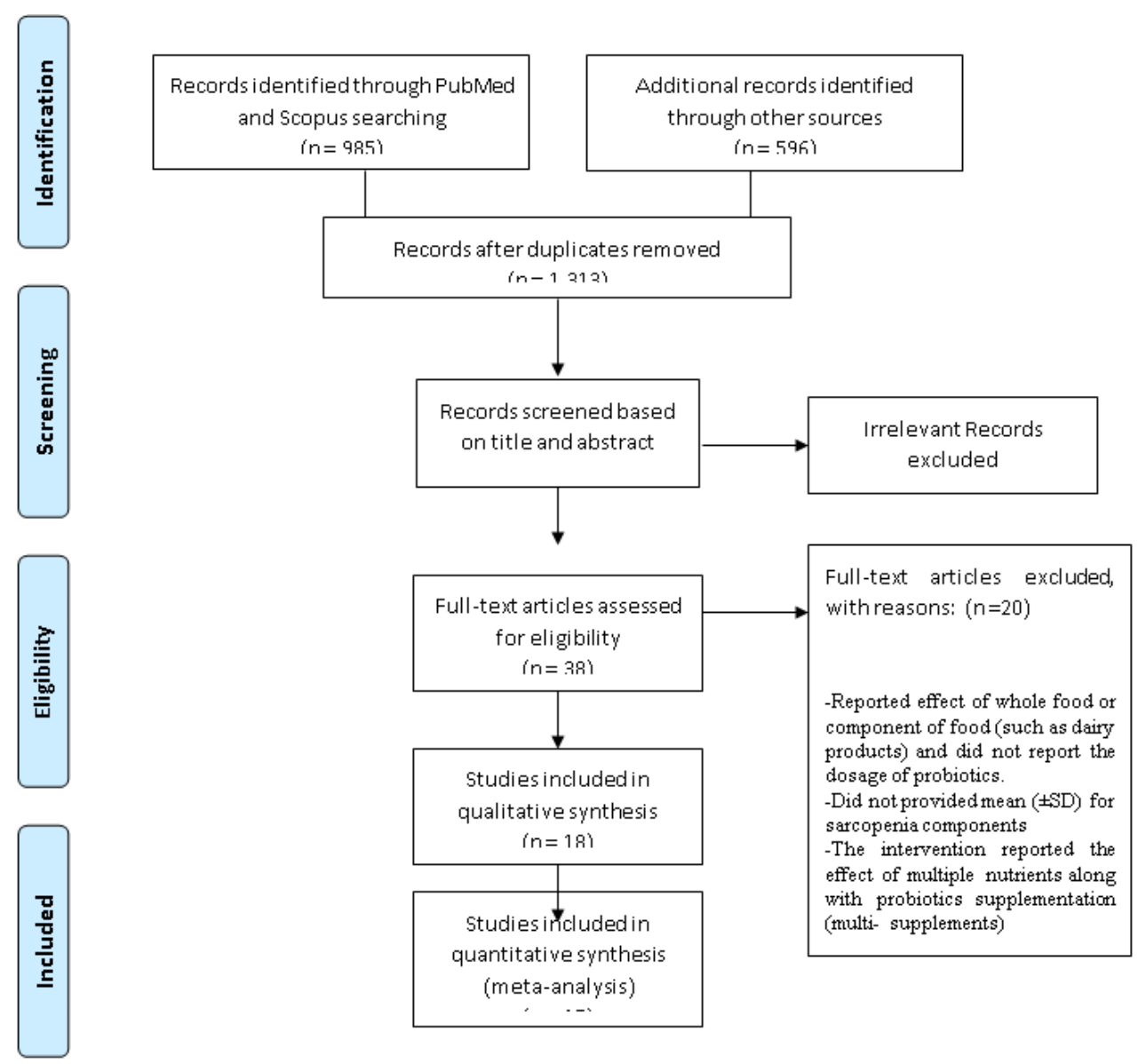

Figure 1

PRISMA flow-chart of study selection process for the impact of probiotic supplements on sarcopenic components in elderly adults. 


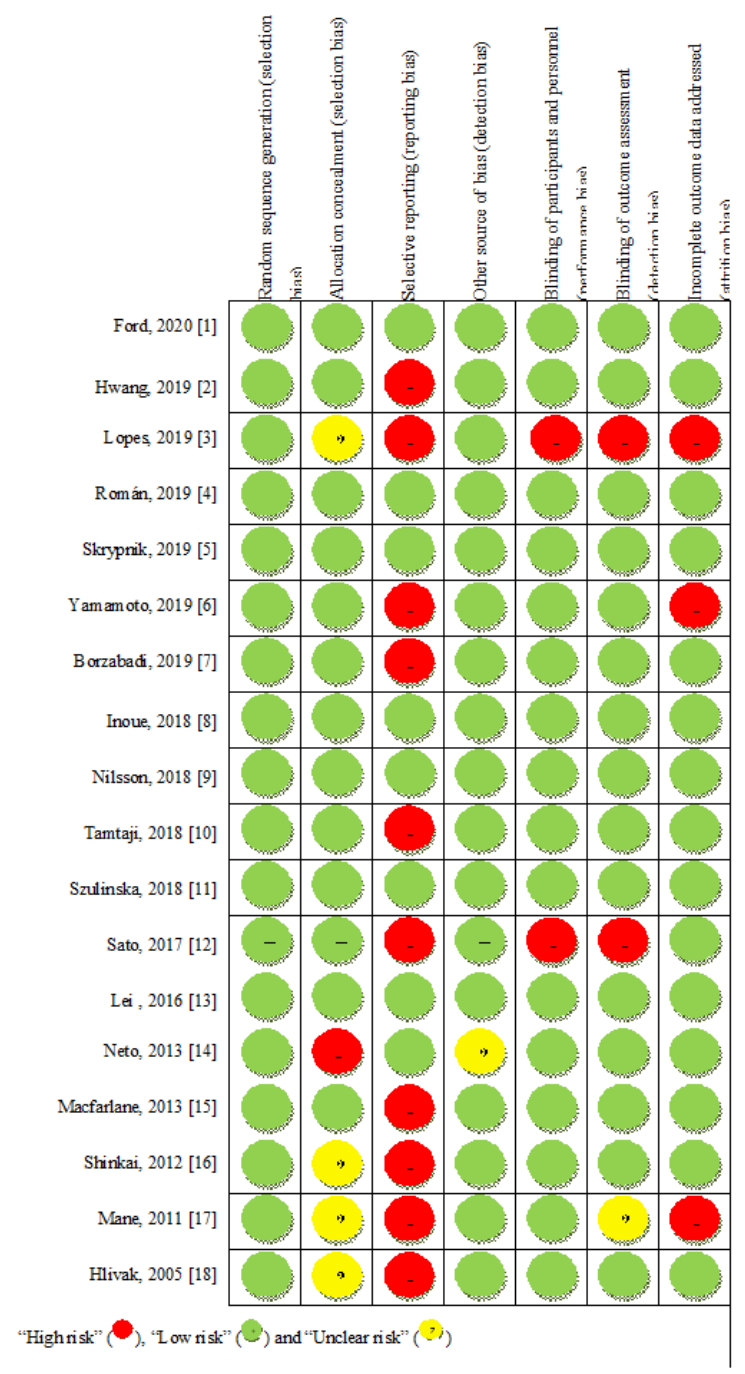

Figure 2

The quality assessment of included studies. 


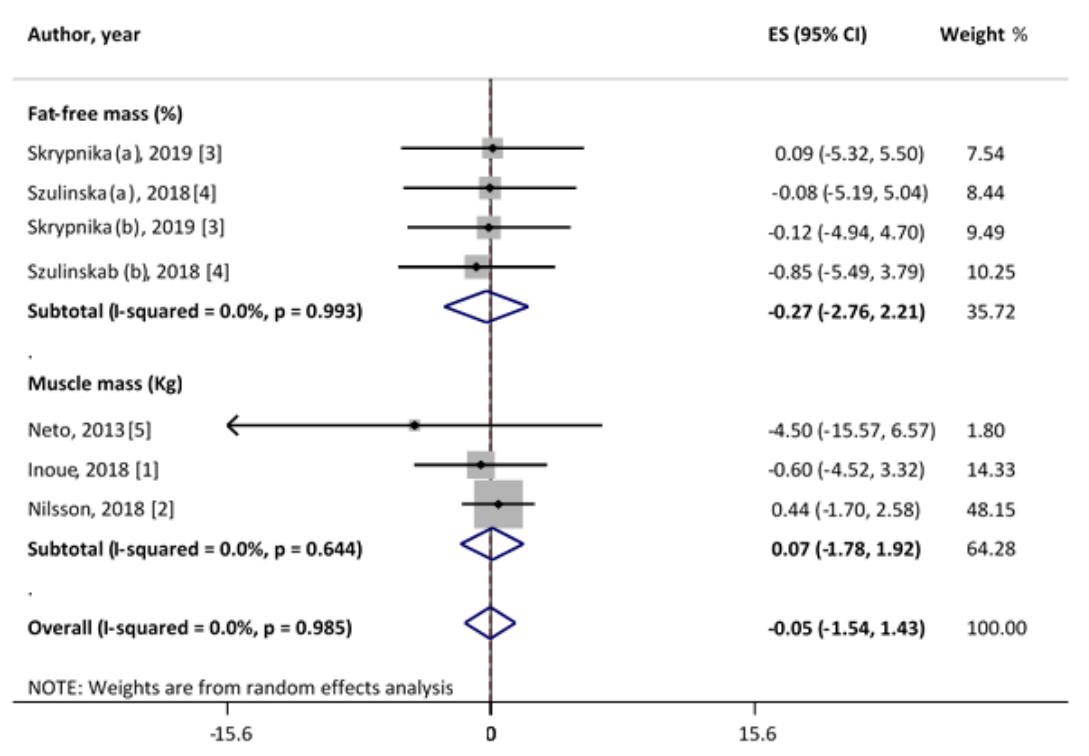

\section{Figure 3}

Forest plot of the effect of probiotic supplementation on fat free mass and muscle mass.

1. Inoue, T., et al., Effect of combined bifidobacteria supplementation and resistance training on cognitive function, body composition and bowel habits of healthy elderly subjects. Benef Microbes, 2018. 9(6): p. 843-853.

2. Nilsson, A.G., et al., Lactobacillus reuteri reduces bone loss in older women with low bone mineral density: a randomized, placebo-controlled, double-blind, clinical trial. J Intern Med, 2018. 284(3): p. 307-317.

3. Skrypnik, K., et al., The effect of multistrain probiotic supplementation in two doses on iron metabolism in obese postmenopausal women: a randomized trial. Food Funct, 2019. 10(8): p. 5228-5238.

4. Szulińska, M., et al., Dose-Dependent Effects of Multispecies Probiotic Supplementation on the Lipopolysaccharide (LPS) Level and Cardiometabolic Profile in Obese Postmenopausal Women: A 12-Week Randomized Clinical Trial. Nutrients, 2018. 10(6).

5. Neto, J.V., C.M. De Melo, and S.M.L. Ribeiro, Effects of three-month intake of synbiotic on inflammation and body composition in the elderly: a pilot study. Nutrients, 2013. 5(4): p. 1276-1286. 


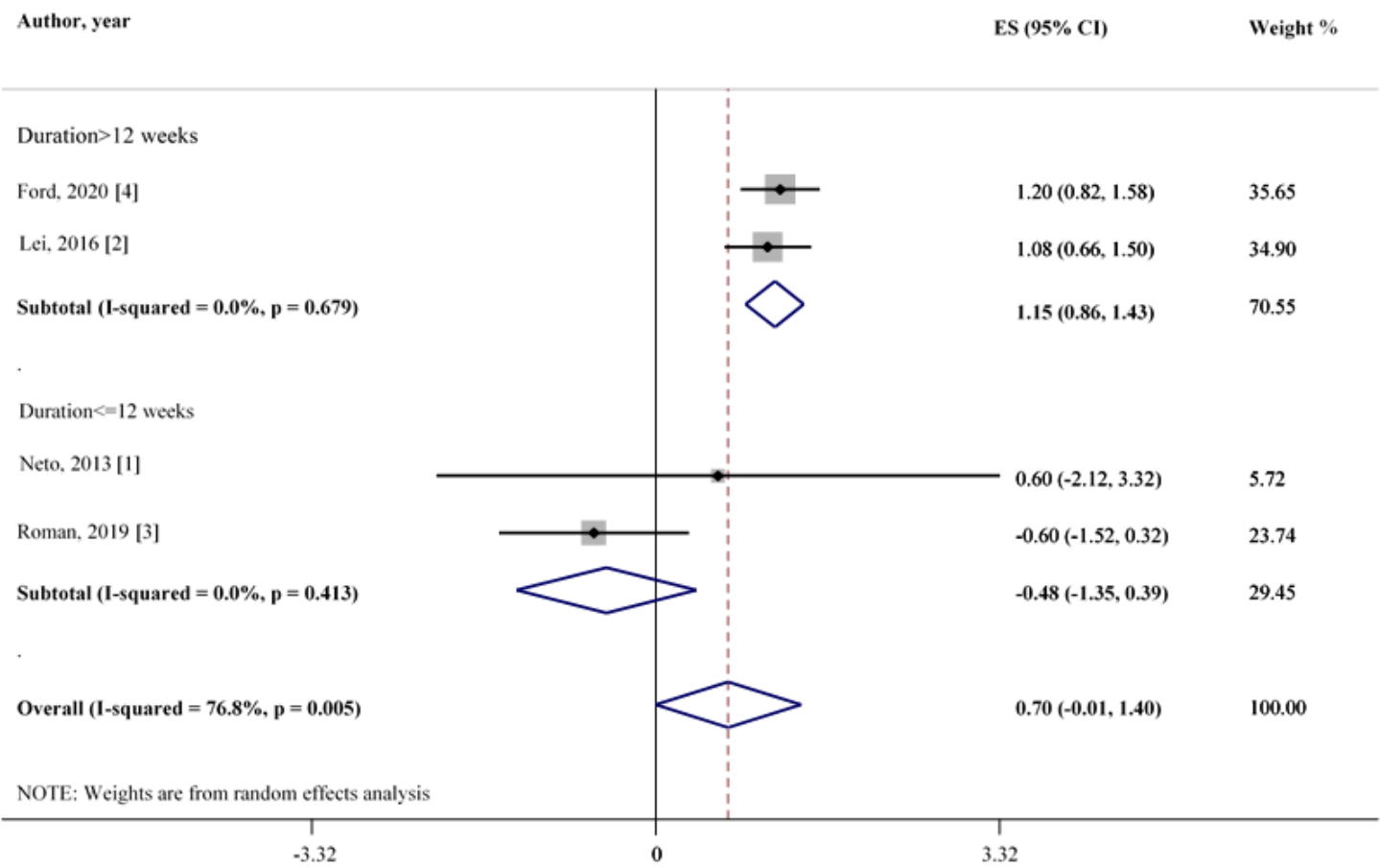

\section{Figure 4}

Forest plot of the effect of probiotic supplementation on muscle strengt

1. Neto, J.V., C.M. De Melo, and S.M.L. Ribeiro, Effects of three-month intake of synbiotic on inflammation and body composition in the elderly: a pilot study. Nutrients, 2013. 5(4): p. 1276-1286.

2. Lei, M., L. Hua, and D. Wang, The effect of probiotic treatment on elderly patients with distal radius fracture: a prospective double-blind, placebocontrolled randomised clinical trial. Beneficial microbes, 2016. 7(5): p. 631-637.

3. Román, E., et al., Effect of a multistrain probiotic on cognitive function and risk of falls in patients with cirrhosis: A randomized trial. Hepatology communications, 2019. 3(5): p. 632-645.

4. Ford, A.L., et al., Microbiota stability and gastrointestinal tolerance in response to a high-protein diet with and without a prebiotic, probiotic, and synbiotic: A randomized, double-blind, placebo-controlled trial in older women. Journal of the Academy of Nutrition and Dietetics, 2020. 120(4): $\mathrm{p}$. 500516. e10.

\section{Supplementary Files}

This is a list of supplementary files associated with this preprint. Click to download.

- Supplementaryfile1.SearchResults.docx

- supplementaryfile2.Listofexcludedstudies.docx 